\title{
Robust Multiple Homography Estimation: An Ill-Solved Problem
}

\author{
Zygmunt L. Szpak, Wojciech Chojnacki, Anton van den Hengel \\ School of Computer Science, The University of Adelaide, SA 5005, Australia \\ \{zygmunt.szpak, wojciech.chojnacki, anton.vandenhengel\}@adelaide.edu.au
}

\begin{abstract}
The estimation of multiple homographies between two piecewise planar views of a rigid scene is often assumed to be a solved problem. We show that contrary to popular opinion various crucial aspects of the task have not been adequately emphasised. We are motivated by a growing body of literature in robust multi-structure estimation that purports to solve the multi-homography estimation problem but in fact does not. We demonstrate that the estimation of multiple homographies is an ill-solved problem by deriving new constraints that a set of mutually compatible homographies must satisfy, and by showing that homographies estimated with prevailing methods fail to satisfy the requisite constraints on real-world data. We also explain why incompatible homographies imply inconsistent epipolar geometries. The arguments and experiments presented in this paper signal the need for a new generation of robust multi-structure estimation methods that have the capacity to enforce constraints on projective entities such as homography matrices.
\end{abstract}

\section{Introduction}

Images in two views of world points lying on a planar surface are related by a homography matrix. Since planar surfaces are ubiquitous in urban environments, estimating multiple homography matrices from image measurements between two views is an important step in many applications such as augmenting reality, stitching and warping images, calibrating cameras, finding a metric reconstruction, and detecting non-rigid motion. Because of the diverse utility of homography matrices, the task of estimating multiple homographies is often used to demonstrate the merits of robust multi-structure estimation methods $[6,7,12,13,19,29,30,34,35]$. In fact, some robust multistructure estimation methods, such as multiRANSAC [39], were specifically designed to address the multi-homography estimation problem. However, an inadvertent oversight has crept into multi-structure estimation methods, one that persists in all state-of-the-art methods that we are familiar with, namely the failure to recognise that a set of homographies that each of these schemes produces is actually not a genuine set of homographies between two views of the same scene. A collection of homography matrices forms a valid set only if the matrices satisfy consistency constraints implied by the rigidity of the motion and the scene. If the constraints are not deliberately enforced, they are not satisfied in typical scenarios. Hence, one of the fundamental problems in estimating multiple homography matrices is to find a way to enforce the consistency constraints-a task reminiscent of that of enforcing the rank-two constraint in the case of the fundamental matrix estimation [17, Sect. 11.1.1].

Explicit formulae for all constraints that must be satisfied have eluded the vision community. It was only as recently as 2011 that a decisive answer pertaining to even just the number of constraints was given [10,11]. Over the years various researchers have managed to identify and enforce a reduced set of constraints. For example, Shashua and Avidan [31] found that homography matrices induced by four or more planes in a 3D scene appearing in two views span a four-dimensional linear subspace. Chen and Suter [5] derived a set of strengthened constraints for the case of three or more homographies in two views. Zelnik-Manor and Irani [36] have shown that another rank-four constraint applies to a set of so-called relative homographies generated by two planes in four or more views. These latter authors also derived constraints for larger sets of homographies and views.

Once isolated, the constraints are typically put to use in a procedure whereby first individual homography matrices are estimated from image data, and then the resulting estimates are upgraded to matrices satisfying the constraints. Following this pattern, Shashua and Avidan as well as Zelnik-Manor and Irani used low-rank approximation under the Frobenius norm to enforce the rank-four constraint. Chen and Suter enforced their set of constraints also via low-rank approximation, but then employed the Mahalanobis norm with covariances of the input homographies. All of these estimation procedures produce matrices that satisfy only incomplete constraints so their true consistency 
cannot be guaranteed.

A few researchers managed to enforce some consistency without appealing to rank constraints. For example, López-Nicolás et al. [22] used the geometry underpinning an epipole constraint, whereas Kirchhof [20] required a fundamental matrix in order to estimate homographies consistently.

Without knowledge of explicit formulae for all of the constraints, it is still possible to implicitly enforce full consistency by recoursing to a parametrisation of the set of all intervening homography matrices. Following this path, Chojnacki et al. [8,9] employed an appropriate parametrisation and a distinct cost function to develop an upgrade procedure based on unconstrained optimisation. Szpak et al. [32] used the same parametrisation and the Sampson distance to develop an alternative technique with a sound statistical basis. Details of this parametrisation will be presented in Section 2.

While a literature review shows that considerable progress has been made on the problem of multihomography estimation in recent years, it is also apparent that many researchers are not aware that bona fide sets of homography matrices need to satisfy constraints. The nescience concerning constraints is particularly evident in the discourse on robust multi-structure estimation. Our contribution, therefore, is two-fold: (1) we demonstrate, from a practical perspective, that failing to enforce consistency constraints on multiple homographies leads to inconsistent estimates of the epipolar geometry between two views, and (2) we derive two new sets of explicit constraints that multiple homographies between two views need to satisfy. Some benefits of having explicit constraints are presented in Section 3 .

\section{Enforcing consistency implicitly}

As mentioned in the introduction, when estimating a set of homographies associated with multiple planes from image correspondences between two views, one must recognise that the homographies involved are interdependent. To get an idea of the relevant dependencies, consider two fixed uncalibrated cameras giving rise to two camera matrices $\mathbf{P}_{1}=\mathbf{K}_{1} \mathbf{R}_{1}\left[\mathbf{I}_{3},-\mathbf{t}_{1}\right]$ and $\mathbf{P}_{2}=\mathbf{K}_{2} \mathbf{R}_{2}\left[\mathbf{I}_{3},-\mathbf{t}_{2}\right]$. Here, the length-3 translation vector $\mathbf{t}_{k}$ and the $3 \times 3$ rotation matrix $\mathbf{R}_{k}$ represent the Euclidean transformation between the $k$-th $(k=1,2)$ camera and the world coordinate system, $\mathbf{K}_{k}$ is a $3 \times 3$ upper triangular calibration matrix encoding the internal parameters of the $k$-th camera, and $\mathbf{I}_{3}$ denotes the $3 \times 3$ identity matrix. Suppose, moreover, that a set of $I$ planes in a 3D scene have been selected. Given $i=1, \ldots, I$, let the $i$-th plane from the collection have a unit outward normal $\mathbf{n}_{i}$ and be situated at a distance $d_{i}$ from the origin of the world coordinate system. Then, for each $i=1, \ldots, I$, the $i$-th plane gives rise to a planar homography between the first and second views described by the $3 \times 3$ matrix

$$
\mathbf{H}_{i}=w_{i} \mathbf{A}+\mathbf{b v}_{i}^{\top},
$$

where

$$
\begin{aligned}
\mathbf{A}=\mathbf{K}_{2} \mathbf{R}_{2} \mathbf{R}_{1}^{-1} \mathbf{K}_{1}^{-1}, & w_{i}=\mathbf{n}_{i}^{\top} \mathbf{t}_{1}-d_{i}, \\
\mathbf{b}=\mathbf{K}_{2} \mathbf{R}_{2}\left(\mathbf{t}_{1}-\mathbf{t}_{2}\right), & \mathbf{v}_{i}=\mathbf{K}_{1}^{-\top} \mathbf{R}_{1} \mathbf{n}_{i} .
\end{aligned}
$$

In the case of calibrated cameras when one may assume that $\mathbf{K}_{1}=\mathbf{K}_{2}=\mathbf{I}_{3}, \mathbf{t}_{1}=\mathbf{0}, \mathbf{R}_{1}=\mathbf{I}_{3}, \mathbf{R}_{2}=\mathbf{R}$, system (2) reduces to

$$
\begin{aligned}
\mathbf{A} & =\mathbf{R}, & w_{i} & =-d_{i}, \\
\mathbf{b} & =\mathbf{t}, & \mathbf{v}_{i} & =\mathbf{n}_{i},
\end{aligned}
$$

with $\mathbf{t}=-\mathbf{R t}_{2}$, and equality (1) becomes the familiar $d i$ rect $n R t$ representation

$$
\mathbf{H}_{i}=-d_{i} \mathbf{R}+\mathbf{t n}_{i}^{\top}
$$

(cf. [1], [25, Sect. 5.3.1]). We stress that all of our subsequent analysis concerns the general uncalibrated case, with $\mathbf{A}, \mathbf{b}, w_{i}$ 's and $\mathbf{v}_{i}$ 's to be interpreted according to (2) rather than (3).

A natural object associated with the matrices $\mathbf{H}_{i}$ is the $3 \times 3 I$ concatenation matrix $\mathbf{H}=\left[\mathbf{H}_{1}, \ldots, \mathbf{H}_{I}\right]$. It proves convenient to consider also the $9 \times I$ matrix $\mathbf{H}=$ $\left[\mathbf{h}_{1}, \ldots \mathbf{h}_{I}\right]$, where, with vec denoting column-wise vectorisation [23], $\mathbf{h}_{i}=\operatorname{vec}\left(\mathbf{H}_{i}\right)$ for each $i=1, \ldots, I$. It turns out that $\mathbf{H}=\mathbf{S T}$, where, with $\otimes$ denoting Kronecker product [23], $\mathbf{S}=\left[\mathbf{I}_{3} \otimes \mathbf{b}, \mathbf{a}\right]$ is a $9 \times 4$ matrix and $\mathbf{T}=\left[\begin{array}{lll}\mathbf{v}_{1} & \ldots & \mathbf{v}_{I} \\ w_{1} & \ldots & w_{I}\end{array}\right]$ is a $4 \times I$ matrix [32]. An immediate consequence of this factorisation is that $\mathbf{H}$ has rank at most four. Whenever $I \geq 5$ the requirement that $\mathbf{H}$ should have rank no greater than four places a genuine constraint on $\mathbf{H}$, and hence also on $\mathbf{H}$. This is the rank-four constraint of Shashua and Avidan mentioned earlier. Since the real $9 \times I$ matrices of rank at most 4 form a manifold of dimension $4(9+I-4)=4 I+20$ [16, Proposition 12.2], the rank-four constraint implies that the dimension of the set of all $\mathbf{H}$ 's is no greater than $4 I+20$ for $I \geq 5$. The ensuing inequality $4 I+20<9 I$ for $I \geq 5$ makes it clear that $\mathbf{H}$ resides in a proper subset of all $3 \times 3 I$ matrices for $I \geq 5$.

The dimensionality count for the H's can be refined and the subsequent conclusions sharpened. Letting $\boldsymbol{\eta}=\left[\mathbf{a}^{\top}, \mathbf{b}^{\top}, \mathbf{v}_{1}^{\top}, \ldots, \mathbf{v}_{I}^{\top}, w_{1}, \ldots, w_{I}\right]^{\top}$ and $\boldsymbol{\Pi}(\boldsymbol{\eta})=$ $\left[\boldsymbol{\Pi}_{1}(\boldsymbol{\eta}), \ldots, \boldsymbol{\Pi}_{I}(\boldsymbol{\eta})\right]$, where $\boldsymbol{\Pi}_{i}(\boldsymbol{\eta})=w_{i} \mathbf{A}+\mathbf{b v}_{i}^{\top}$ for each $i=1, \ldots, I, \mathbf{H}$ can be represented as

$$
\mathbf{H}=\boldsymbol{\Pi}(\boldsymbol{\eta})
$$

In this formulation, $\boldsymbol{\eta}$ appears as the vector of latent variables that link all the constituent matrices together and provide a natural parametrisation of the set of all H's. Since $\boldsymbol{\eta}$ has a total of $4 I+12$ entries, the totality of all matrices 
of the form $\boldsymbol{\Pi}(\boldsymbol{\eta})$ has dimension no greater than $4 I+12$. Here, the relevant notion of dimension is that of dimension of a semi-algebraic set. A semi-algebraic set is a finite union of subsets of some ambient real $n$-dimensional space $\mathbb{R}^{n}$, each defined as a solution set of a finite system of polynomial equations and inequalities. Any semialgebraic set is locally, on a dense subset, a submanifold embedded in the ambient space. The dimension of a semialgebraic set is the largest dimension at points around which the set is a submanifold. The set of all $\boldsymbol{\Pi}(\boldsymbol{\eta})$ 's is semialgebraic - it is the image of $\mathbb{R}^{4 I+12}$ by the polynomial map $\boldsymbol{\eta} \mapsto \boldsymbol{\Pi}(\boldsymbol{\eta})$ (a polynomial map is one whose all components are polynomials in the arguments), and, according to the Tarski-Seidenberg theorem, for any polynomial map $f: \mathbb{R}^{m} \rightarrow \mathbb{R}^{n}$, the image of $\mathbb{R}^{m}$ by $f$ is a semi-algebraic [4, Proposition 2.2.7]. With the aid of a rather subtle argument, the dimension of the set of all $\boldsymbol{\Pi}(\boldsymbol{\eta})$ 's can be calculated exactly, and it is found to be equal to $4 I+7[10,11]$. Since $4 I+7<9 I$ whenever $I \geq 2$, it follows that $\mathbf{H}$ resides in a proper subset of all $3 \times 3 I$ matrices for $I \geq 2$. This is an improvement over the previous similar statement which was valid only for $I \geq 5$. It is now clear that the requirement that $\mathbf{H}$ take the form as per (4) whenever $I \geq 2$ can be seen as an implicit constraint on $\mathbf{H}$, with the consequence that the $\mathbf{H}_{i}$ 's are all interdependent.

The parametrisation (4) gives an effective handle on the inter-dependencies in the matrices $\mathbf{H}_{i}$. Note that this parametrisation is not minimal in that if $\mathbf{H}=\boldsymbol{\Pi}(\boldsymbol{\eta})$ holds for a particular vector $\boldsymbol{\eta}$, then we also have $\mathbf{H}=\boldsymbol{\Pi}\left(\boldsymbol{\eta}^{\prime}\right)$ for any vector $\boldsymbol{\eta}^{\prime}$ of the form

$$
\begin{aligned}
\boldsymbol{\eta}^{\prime}= & {\left[\operatorname{vec}\left(\beta \mathbf{A}+\mathbf{b c}^{\top}\right), \alpha \mathbf{b}, \alpha^{-1} \mathbf{v}_{1}-\alpha^{-1} \beta^{-1} \mathbf{c}, \ldots,\right.} \\
& \left.\alpha^{-1} \mathbf{v}_{I}-\alpha^{-1} \beta^{-1} \mathbf{c}, \beta^{-1} w_{1}, \ldots, \beta^{-1} w_{I}\right]^{\top}
\end{aligned}
$$

where $\alpha$ and $\beta$ are non-zero numbers, and $\mathbf{c}$ is a length-3 vector. The arbitrariness in the choice of $\alpha, \beta$, and $\mathbf{c}$ reflects five degrees of parametrisation gauge freedom. In particular, if $\mathbf{H}=\boldsymbol{\Pi}(\boldsymbol{\eta})$ holds for a particular vector $\boldsymbol{\eta}$, then it is not true that the entries $\boldsymbol{\eta}$ have to be necessarily interpreted as ones satisfying (2) for the underlying values of $\mathbf{K}_{k}, \mathbf{R}_{k}, \mathbf{t}_{k}, d_{i}$, and $\mathbf{n}_{i}(k=1,2, i=1, \ldots, I)$. It is also worth stressing that the fact that the parametrisation (4) is not minimal is not a limitation-on the contrary, the parametrisation is perfectly suited to the purpose of parameter estimation, as it enforces all underlying constraints on the $\mathbf{H}_{i}$ 's.

A further benefit of the parametrisation (4) is that it automatically yields the expression for the genuine rank-2 fundamental matrix linking the two views, namely

$$
\mathbf{F}=[\mathbf{b}]_{\times} \mathbf{A} .
$$

Here, for a length-3 vector $\mathbf{a}=\left[a_{1}, a_{2}, a_{3}\right]^{\top},[\mathbf{a}]_{\times}$denotes the $3 \times 3$ anti-symmetric matrix defined by

$$
[\mathbf{a}]_{\times}=\left[\begin{array}{ccc}
0 & -a_{3} & a_{2} \\
a_{3} & 0 & -a_{1} \\
-a_{2} & a_{1} & 0
\end{array}\right]
$$

The fundamental matrix given in (5) is fully compatible with the homography matrices $\mathbf{H}_{i}$ in that it satisfies the relations $\mathbf{H}_{i}^{\top} \mathbf{F}+\mathbf{F}^{\top} \mathbf{H}_{i}=\mathbf{0}$ for $i=1, \ldots, I$ (cf. [17, Sect. 13.1.1]). Moreover, the expression (5) is invariant, up to a scalar factor, to any change $\boldsymbol{\eta} \mapsto \boldsymbol{\eta}^{\prime}$ related to the parametrisation gauge freedom.

\section{Benefits of explicit constraints}

Despite the numerous aforementioned advantages, the use of latent variables to implicitly enforce consistency constraints also has some notable drawbacks. The latent variable method does not provide a means to directly measure the extent to which a collection of homography matrices are compatible. Furthermore, finding suitable initial values for the latent variables is a non-trivial task. The initialisation methods described by Chojnacki et al. [8,9] and Szpak et al. [32] are based on factorising a collection of homography matrices. The factorisation methods are sensitive to noise and are not robust to outliers. This implies that if one of the homography matrices is a gross outlier, the initial values for the latent variables will be poor, and the subsequent non-linear optimisation may find a poor minimum.

Knowledge of explicit formulae for homography constraints would be advantageous for several reasons: (1) it would give rise to novel homography estimation methods that enforce full compatibility without recourse to latent variables; (2) it would spur the development of new global optimisation methods for multi-homography estimation, analogous to what has recently been achieved for fundamental matrix estimation [37,38]; and (3) it would lead to a new generation of robust multi-structure fitting methods that, unlike existing methods, yield estimates with consistent epipolar geometry.

In this paper we take a step toward achieving the goal of determining all homography constraints. We derive two new sets of consistency constraints and use them to measure, for the first time, the extent to which separately estimated homographies are mutually incompatible. Our findings suggest that none of the state-of-the-art multi-structure estimation methods adequately address the problem of multiple homography estimation, because none of them enforce consistency constraints. Consequently, robust multiple homography estimation continues to be an ill-solved problem.

To make the derivation of the constraints more accessible, we collect in the next section some necessary technical prerequisites. 


\section{Algebraic prerequisites}

Let $\mathbf{A}$ and $\mathbf{B}$ be two $3 \times 3$ matrices. The linear matrix pencil of the matrix pair $(\mathbf{A}, \mathbf{B})$ is the matrix function $\lambda \mapsto \mathbf{A}-\lambda \mathbf{B}$. The characteristic polynomial of $(\mathbf{A}, \mathbf{B})$, $p_{\mathbf{A}, \mathbf{B}}$, is defined by $p_{\mathbf{A}, \mathbf{B}}(\lambda)=\operatorname{det}(\mathbf{A}-\lambda \mathbf{B})$. When $\mathbf{A}$ and $\mathbf{B}$ are represented as $\mathbf{A}=\left[\mathbf{a}_{1}, \mathbf{a}_{2}, \mathbf{a}_{3}\right]$ and $\mathbf{B}=\left[\mathbf{b}_{1}, \mathbf{b}_{2}, \mathbf{b}_{3}\right]$ with $\mathbf{a}_{i}$ and $\mathbf{b}_{i}(i=1,2,3)$ being length- 3 vectors, the characteristic polynomial $p_{\mathbf{A}, \mathbf{B}}$ can be explicitly written as $p_{\mathbf{A}, \mathbf{B}}(\lambda)=\sum_{i=0}^{3}(-1)^{i} c_{i} \lambda^{i}$, where

$c_{0}=\operatorname{det} \mathbf{A}$,

$c_{1}=\operatorname{det}\left(\left[\mathbf{b}_{1}, \mathbf{a}_{2}, \mathbf{a}_{3}\right]\right)+\operatorname{det}\left(\left[\mathbf{a}_{1}, \mathbf{b}_{2}, \mathbf{a}_{3}\right]\right)+\operatorname{det}\left(\left[\mathbf{a}_{1}, \mathbf{a}_{2}, \mathbf{b}_{3}\right]\right)$,

$c_{2}=\operatorname{det}\left(\left[\mathbf{a}_{1}, \mathbf{b}_{2}, \mathbf{b}_{3}\right]\right)+\operatorname{det}\left(\left[\mathbf{b}_{1}, \mathbf{a}_{2}, \mathbf{b}_{3}\right]\right)+\operatorname{det}\left(\left[\mathbf{b}_{1}, \mathbf{b}_{2}, \mathbf{a}_{3}\right]\right)$,

$c_{3}=\operatorname{det} \mathbf{B}$.

The characteristic polynomial arises in connection with the generalised eigenvalue problem

$$
\mathbf{A x}=\lambda \mathbf{B} \mathbf{x} .
$$

As with the standard eigenvalue problem, eigenvalues for the problem (6) occur precisely where the matrix pencil $\mathbf{A}-$ $\lambda \mathbf{B}$ is singular. In other words, the eigenvalues of $(\mathbf{A}, \mathbf{B})$ are the roots of $p_{\mathbf{A}, \mathbf{B}}$.

Suppose that the generalised eigenvalue problem (6) has a double eigenvalue $\mu$, that is, there exist linearly independent length- 3 vectors $\mathbf{v}_{1}$ and $\mathbf{v}_{2}$ such that $\mathbf{A} \mathbf{v}_{i}=\mu \mathbf{B} \mathbf{v}_{i}$ for $i=1,2$. Then, as it turns out, $\mathbf{A}$ and $\mathbf{B}$ have to necessarily satisfy a certain algebraic constraint. This constraint is expressed as the vanishing of a homogeneous quartic polynomial in the entries of $\mathbf{A}$ and $\mathbf{B}$.

To reveal the constraint, we first observe that $\mu$ is a double root of $p_{\mathbf{A}, \mathbf{B}}$. Indeed, let $\mathbf{v}_{3}$ be an arbitrary length-3 vector not belonging to the linear span of $\mathbf{v}_{1}$ and $\mathbf{v}_{2}$; for example, we may assume that $\mathbf{v}_{3}=\mathbf{v}_{1} \times \mathbf{v}_{2}$. Then $\mathbf{v}_{1}, \mathbf{v}_{2}$, and $\mathbf{v}_{3}$ form a basis for $\mathbb{R}^{3}$, and hence the matrix $\mathbf{S}=\left[\mathbf{v}_{1}, \mathbf{v}_{2}, \mathbf{v}_{3}\right]$ is non-singular. Let $\tilde{\mathbf{A}}=\mathbf{S}^{-1} \mathbf{A S}$ and $\tilde{\mathbf{B}}=\mathbf{S}^{-1} \mathbf{B S}$. With $\mathbf{e}_{i}(i=1,2,3)$ being the standard unit vectors in $\mathbb{R}^{3}$, we have $\mathbf{v}_{i}=\mathbf{S e}_{i}$ for $i=1,2,3$. It is immediate that, for $i=1,2, \tilde{\mathbf{A}} \mathbf{e}_{i}=\mu \tilde{\mathbf{B}} \mathbf{e}_{i}$ and so $(\tilde{\mathbf{A}}-\lambda \tilde{\mathbf{B}}) \mathbf{e}_{i}=(\mu-\lambda) \tilde{\mathbf{B}} \mathbf{e}_{i}$. Hence the pencil $\tilde{\mathbf{A}}-\lambda \tilde{\mathbf{B}}$ takes the form

$$
\tilde{\mathbf{A}}-\lambda \tilde{\mathbf{B}}=\left[\begin{array}{lll}
(\mu-\lambda) \tilde{b}_{11} & (\mu-\lambda) \tilde{b}_{12} & \tilde{a}_{13}-\lambda \tilde{b}_{13} \\
(\mu-\lambda) \tilde{b}_{21} & (\mu-\lambda) \tilde{b}_{22} & \tilde{a}_{23}-\lambda \tilde{b}_{23} \\
(\mu-\lambda) \tilde{b}_{31} & (\mu-\lambda) \tilde{b}_{32} & \tilde{a}_{33}-\lambda \tilde{b}_{33}
\end{array}\right]
$$

and we have

$$
\begin{aligned}
p_{\tilde{\mathbf{A}}, \tilde{\mathbf{B}}}(\lambda) & =\left|\begin{array}{lll}
(\mu-\lambda) \tilde{b}_{11} & (\mu-\lambda) \tilde{b}_{12} & \tilde{a}_{13}-\lambda \tilde{b}_{13} \\
(\mu-\lambda) \tilde{b}_{21} & (\mu-\lambda) \tilde{b}_{22} & \tilde{a}_{23}-\lambda \tilde{b}_{23} \\
(\mu-\lambda) \tilde{b}_{31} & (\mu-\lambda) \tilde{b}_{32} & \tilde{a}_{33}-\lambda \tilde{b}_{33}
\end{array}\right| \\
& =(\mu-\lambda)^{2}\left|\begin{array}{lll}
\tilde{b}_{11} & \tilde{b}_{12} & \tilde{a}_{13}-\lambda \tilde{b}_{13} \\
\tilde{b}_{21} & \tilde{b}_{22} & \tilde{a}_{23}-\lambda \tilde{b}_{23} \\
\tilde{b}_{31} & \tilde{b}_{32} & \tilde{a}_{33}-\lambda \tilde{b}_{33}
\end{array}\right|,
\end{aligned}
$$

which shows that $p_{\tilde{\mathbf{A}}, \tilde{\mathbf{B}}}$ has double root $\mu$. But $p_{\tilde{\mathbf{A}}, \tilde{\mathbf{B}}}$ coincides with $p_{\mathbf{A}, \mathbf{B}}$, given that

$$
\begin{aligned}
p_{\tilde{\mathbf{A}}, \tilde{\mathbf{B}}}(\lambda) & \left.=\operatorname{det}\left(\mathbf{S}^{-1}\left(\mathbf{A}-\lambda \mathbf{B}_{3}\right) \mathbf{S}\right)\right) \\
& =\operatorname{det}\left(\mathbf{S}^{-1}\right) \operatorname{det}\left(\mathbf{A}-\lambda \mathbf{B}_{3}\right) \operatorname{det}(\mathbf{S}) \\
& =\operatorname{det}(\mathbf{S})^{-1} \operatorname{det}\left(\mathbf{A}-\lambda \mathbf{I}_{3}\right) \operatorname{det}(\mathbf{S})=p_{\mathbf{A}, \mathbf{B}}(\lambda) .
\end{aligned}
$$

Therefore, $p_{\mathbf{A}, \mathbf{B}}$ has a fortiori double root $\mu$.

Now, let $p(x)=a x^{3}+b x^{2}+c x+d$ be a general cubic polynomial with real coefficients. The discriminant of $p$, $\Delta(p)$, is given by

$$
\Delta(p)=18 a b c d-4 b^{3} d+b^{2} c^{2}-4 a c^{3}-27 a^{2} d^{2} .
$$

A fundamental property of the discriminant is that if $p$ has a double root, then $\Delta(p)=0$ [18, Sect. 10.3].

In light of the above, if the problem (6) has a double eigenvalue, then the entries of $\mathbf{A}$ and $\mathbf{B}$ satisfy the constraint

$$
\Delta\left(p_{\mathbf{A}, \mathbf{B}}\right)=0 .
$$

\section{New consistency constraints}

Let $i$ and $j$ be two different elements of $\{1, \ldots, I\}$ and consider the corresponding homography matrices

$$
\mathbf{H}_{i}=w_{i} \mathbf{A}+\mathbf{b v}_{i}^{\top} \quad \text { and } \quad \mathbf{H}_{j}=w_{j} \mathbf{A}+\mathbf{b} \mathbf{v}_{j}^{\top} .
$$

Taking into account that $\mathbf{v}_{i}^{\top}\left(\mathbf{v}_{i} \times \mathbf{v}_{j}\right)=\mathbf{v}_{j}^{\top}\left(\mathbf{v}_{i} \times \mathbf{v}_{j}\right)=0$, we see that $\mathbf{H}_{i}\left(\mathbf{v}_{i} \times \mathbf{v}_{j}\right)=w_{i} \mathbf{A}\left(\mathbf{v}_{i} \times \mathbf{v}_{j}\right)$ and $\mathbf{H}_{j}\left(\mathbf{v}_{i} \times\right.$ $\left.\mathbf{v}_{j}\right)=w_{j} \mathbf{A}\left(\mathbf{v}_{i} \times \mathbf{v}_{j}\right)$. Hence

$$
\mathbf{H}_{i}\left(\mathbf{v}_{i} \times \mathbf{v}_{j}\right)=w_{i} w_{j}^{-1} \mathbf{H}_{j}\left(\mathbf{v}_{i} \times \mathbf{v}_{j}\right),
$$

so $w_{i} w_{j}^{-1}$ is an eigenvalue of the generalised eigenvalue problem

$$
\mathbf{H}_{i} \mathbf{x}=\lambda \mathbf{H}_{j} \mathbf{x} .
$$

Note that if $\mathbf{c}$ is any length-3 vector, then (7) holds with $\mathbf{A}$ replaced by $\mathbf{A}+\mathbf{b c}^{\top}, \mathbf{b v}_{i}^{\top}$ replaced by $\mathbf{b}\left(\mathbf{v}_{i}-w_{i} \mathbf{c}\right)^{\top}$, and $\mathbf{b v}_{j}^{\top}$ replaced by $\mathbf{b}\left(\mathbf{v}_{j}-w_{j} \mathbf{c}\right)^{\top}$. Accordingly, (8) holds with $\left(\mathbf{v}_{i}-w_{i} \mathbf{c}\right) \times\left(\mathbf{v}_{j}-w_{j} \mathbf{c}\right)$ substituted for $\mathbf{v}_{i} \times \mathbf{v}_{j}$. Now, as $\mathbf{c}$ varies, the vectors

$$
\left(\mathbf{v}_{i}-w_{i} \mathbf{c}\right) \times\left(\mathbf{v}_{j}-w_{j} \mathbf{c}\right)=\left(\mathbf{c}-w_{i}^{-1} \mathbf{v}_{i}\right) \times\left(w_{j} \mathbf{v}_{i}-w_{i} \mathbf{v}_{j}\right)
$$

fill out a two-dimensional linear space, namely the space of all length- 3 vectors orthogonal to $w_{j} \mathbf{v}_{i}-w_{i} \mathbf{v}_{j}$. Thus $w_{i} w_{j}^{-1}$ is in fact a double eigenvalue for the problem (9). Using the material from Section 4, we conclude that

$$
\Delta\left(p_{\mathbf{H}_{i}, \mathbf{H}_{j}}\right)=0 .
$$

A closer inspection reveals that the expression on the lefthand side is a homogeneous polynomial of degree 12 . 


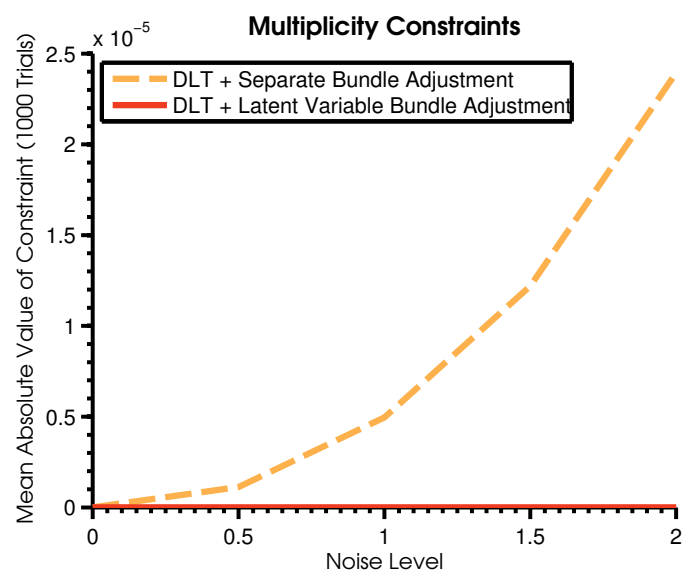

(a)

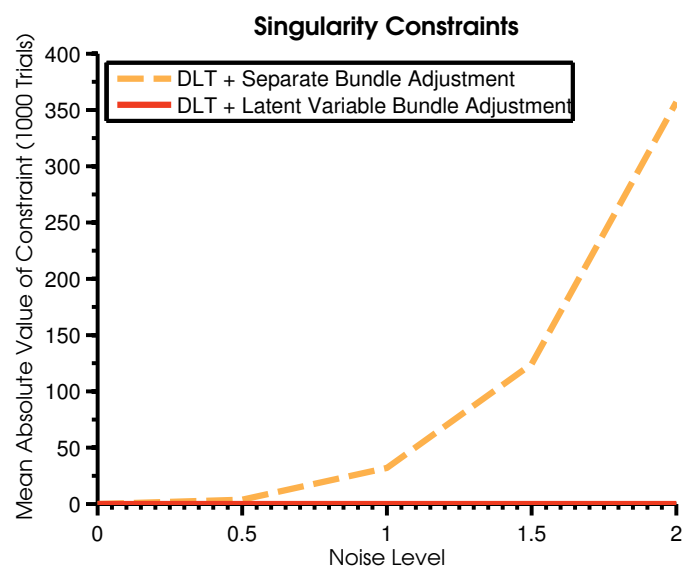

(b)

Figure 1: Evaluation of homography compatibility constraints on synthetic data. Numerous random piecewise planar scenes were generated, and independent Gaussian noise was added to corresponding points between two views of the synthetic scene. Two sets of homography matrices were generated. The first set consisted of homography matrices that were estimated using DLT and refined with bundle adjustment based on the latent variable parametrisation of Szpak et al. [32] which guarantees consistency. The second set consisted of homographies that were estimated using DLT and refined with separate bundle adjustment. Assessment of the multiplicity (panel (a)) and the singularity (panel (b)) constraints on the first set of homographies served to confirm the validity of our formulae - the constraints were satisfied even when the noise level was varied. In contrast, evaluation of the constraints on the second set of homographies revealed that consistency constraints were progressively violated as the noise level was increased. The experiments demonstrate that separate homography estimation results in incompatible homographies.

Equations (10) corresponding to all pairs $(i, j)$ with $i \neq$ $j$ form the first of our two new sets of consistency constraints. We shall refer to this set of constraints as the multiplicity constraints. This set comprises $\left(\begin{array}{l}I \\ 2\end{array}\right)=I(I-1) / 2$ elements. Since $I(I-1) / 2>5 I-7$ whenever $I \geq 10$, with $5 I-7=9 I-(4 I+7)$ being the cardinality of a full set of constraints, we see that the new constraints are not entirely functionally independent at least when $I \geq 10$. Unlike the rank-four constraint, the constraints (10) apply for all values of $I$ no smaller than 2 .

Note that if $\lambda_{1}, \ldots, \lambda_{I}$ are non-zero scalars, then

$$
\Delta\left(p_{\lambda_{i} \mathbf{H}_{i}, \lambda_{j} \mathbf{H}_{j}}\right)=\lambda_{i}^{6} \lambda_{j}^{6} \Delta\left(p_{\mathbf{H}_{i}, \mathbf{H}_{j}}\right) .
$$

This identity reveals that the vanishing of $\Delta\left(p_{\left.\lambda_{i} \mathbf{H}_{i}, \lambda_{j} \mathbf{H}_{j}\right)}\right.$ for any pair $(i, j)$ with $i \neq j$ is equivalent to the vanishing of $\Delta\left(p_{\mathbf{H}_{i}, \mathbf{H}_{j}}\right)$. Thus equations (10) corresponding to all pairs $(i, j)$ with $i \neq j$ are genuine constraints on the homographies represented by the matrices $\mathbf{H}_{i}$.

The two-dimensional eigenspace for the problem (9) with corresponding double eigenvalue $w_{i} w_{j}^{-1}$ has a natural geometric meaning. Indeed, one verifies directly that

$$
\mathbf{H}_{j}^{-1} \mathbf{H}_{i}=\frac{w_{i}}{w_{j}}\left(\mathbf{I}_{3}+\frac{1}{w_{i}\left(\mathbf{v}_{j}^{\top} \mathbf{A}^{-1} \mathbf{b}+w_{j}\right)} \mathbf{A}^{-1} \mathbf{b}\left(w_{j} \mathbf{v}_{i}-w_{i} \mathbf{v}_{j}\right)^{\top}\right) .
$$

It follows from this formula that if $\mathbf{a}_{1}$ and $\mathbf{a}_{2}$ are two linearly independent length- 3 vectors orthogonal to $w_{j} \mathbf{v}_{i}-$ $w_{i} \mathbf{v}_{j}$, then $\mathbf{a}_{1}$ and $\mathbf{a}_{2}$ are eigenvectors of $\mathbf{H}_{j}^{-1} \mathbf{H}_{i}$ with corresponding eigenvalue $w_{i} w_{j}^{-1}$. In other words, the twodimensional linear space spanned by $\mathbf{a}_{1}$ and $\mathbf{a}_{2}$, denoted by $\operatorname{lin}\left\{\mathbf{a}_{1}, \mathbf{a}_{2}\right\}$, is the eigenspace of $\mathbf{H}_{j}^{-1} \mathbf{H}_{i}$ corresponding to the (double) eigenvalue $w_{i} w_{j}^{-1}$. In addition, $\mathbf{A}^{-1} \mathbf{b}$ is an eigenvector of $\mathbf{H}_{j}^{-1} \mathbf{H}_{i}$ corresponding to the eigenvalue

$$
\lambda_{i j}=\left(w_{i}+\mathbf{v}_{i}^{\top} \mathbf{A}^{-1} \mathbf{b}\right)\left(w_{j}+\mathbf{v}_{j}^{\top} \mathbf{A}^{-1} \mathbf{b}\right)^{-1} .
$$

Consequently, $\mathbf{H}_{j}^{-1} \mathbf{H}_{i}$ is a matrix of a planar homology with a line of fixed points (called the axis), each represented by a one-dimensional linear subspace of $\operatorname{lin}\left\{\mathbf{a}_{1}, \mathbf{a}_{2}\right\}$, and a fixed point (called the vertex), not on the line, represented by the scalar multiples of $\mathbf{A}^{-1} \mathbf{b}$. As $\mathbf{A}^{-1} \mathbf{b}=$ $\mathbf{K}_{1} \mathbf{R}_{1}\left(\mathbf{t}_{1}-\mathbf{t}_{2}\right)=-\mathbf{P}_{1} \mathbf{c}_{2}$, where $\mathbf{c}_{2}=\left[\mathbf{t}_{2}^{\top}, 1\right]^{\top}$ is the projection centre of the second camera (satisfying $\mathbf{P}_{2} \mathbf{c}_{2}=\mathbf{0}$ ), we see that $\mathbf{A}^{-1} \mathbf{b}$ represents the epipole in the first image. It is clear that the eigenspace for the problem (9) corresponding to the double eigenvalue $w_{i} w_{j}^{-1}$ is identical with $\operatorname{lin}\left\{\mathbf{a}_{1}, \mathbf{a}_{2}\right\}$. Consequently, this eigenspace can be geometrically interpreted as the axis of the homology $\mathbf{H}_{j}^{-1} \mathbf{H}_{i}$.

Interestingly, the analysis in the last paragraph leads to yet other consistency constraints in the case where $I \geq 3$. Assuming that $I \geq 3$, let $(i, j)$ and $(k, l)$ be two different pairs of elements of $\{1, \ldots, I\}$ such that $i \neq j$ and $k \neq l$. Then $\mathbf{H}_{j}^{-1} \mathbf{H}_{i} \mathbf{e}=\lambda_{i j} \mathbf{e}$ and $\mathbf{H}_{l}^{-1} \mathbf{H}_{k} \mathbf{e}=\lambda_{k l} \mathbf{e}$, where $\mathbf{e}$ is short for $\mathbf{A}^{-1} \mathbf{b}$. Consequently, for any two natural numbers $n$ and $m$,

$$
\left(\mathbf{H}_{j}^{-1} \mathbf{H}_{i}\right)^{n}\left(\mathbf{H}_{l}^{-1} \mathbf{H}_{k}\right)^{m} \mathbf{e}=\left(\mathbf{H}_{l}^{-1} \mathbf{H}_{k}\right)^{m}\left(\mathbf{H}_{j}^{-1} \mathbf{H}_{i}\right)^{n} \mathbf{e}=\lambda_{i j}^{n} \lambda_{k l}^{m} \mathbf{e}
$$

and so

$$
\left[\left(\mathbf{H}_{j}^{-1} \mathbf{H}_{i}\right)^{n},\left(\mathbf{H}_{l}^{-1} \mathbf{H}_{k}\right)^{m}\right] \mathbf{e}=\mathbf{0},
$$




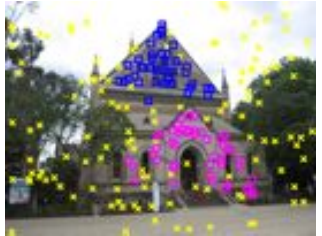

(a) elderhalla

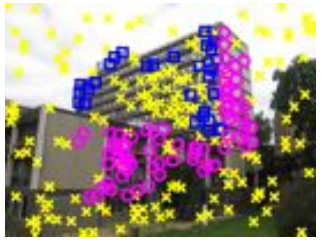

(f) napiera

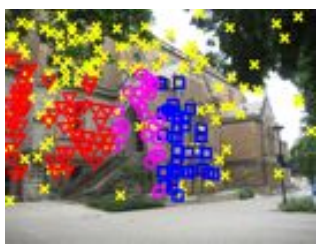

(k) elderhallb

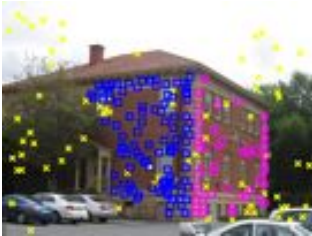

(b) ladysymon

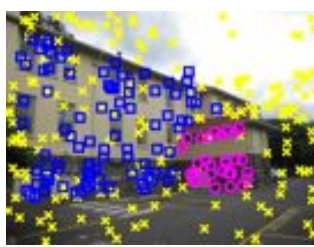

(g) hartley

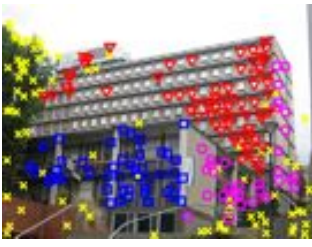

(1) napierb

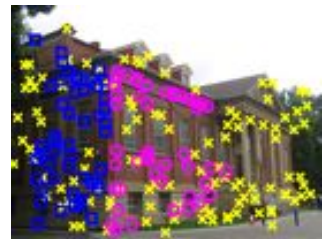

(c) library

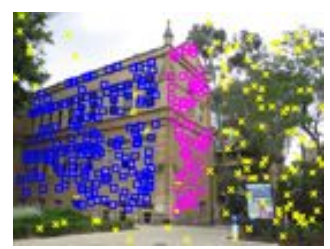

(h) oldclassicswing

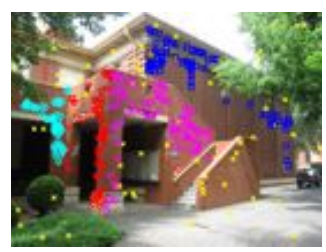

(m) johnsona

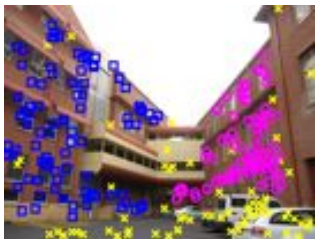

(d) nese

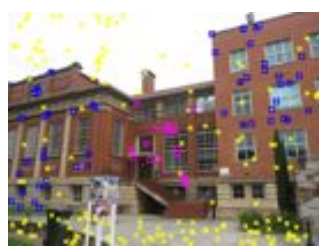

(i) barrsmith

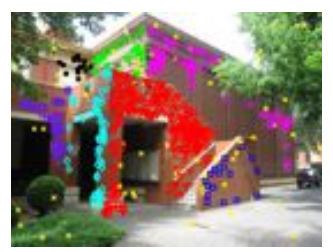

(n) johnsonb

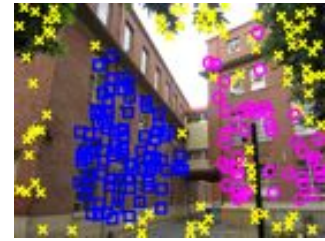

(e) sene

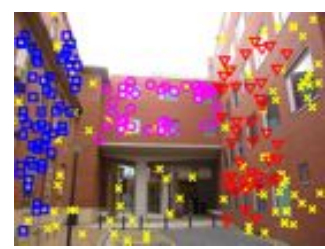

(j) neem

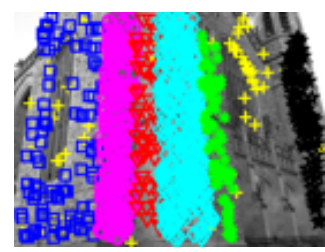

(o) bonhall

Figure 2: AdelaideRMF dataset consisting of image pairs and manually labelled keypoint correspondences which were obtained by SIFT matching. Yellow markers denote outliers (random incorrect matches), while all other coloured markers denote groups of points that can be associated with planar surfaces in three-dimensional space.

where, for any square matrices $\mathbf{S}$ and $\mathbf{T},[\mathbf{S}, \mathbf{T}]=\mathbf{S T}-\mathbf{T S}$. It follows that $\left[\left(\mathbf{H}_{j}^{-1} \mathbf{H}_{i}\right)^{n},\left(\mathbf{H}_{l}^{-1} \mathbf{H}_{k}\right)^{m}\right]$ is singular,

$$
\operatorname{det}\left[\left(\mathbf{H}_{j}^{-1} \mathbf{H}_{i}\right)^{n},\left(\mathbf{H}_{l}^{-1} \mathbf{H}_{k}\right)^{m}\right]=0 .
$$

With $i, j, k, l, n$, and $m$ assuming various respective values, equations (11) give additional consistency constraints. To obtain a finite, manageable set of constraints, it is practical to assume that $n$ and $m$ run independently over the limited set $\{1,2,3\}$. Like in the case of equations (10), equations (11) are constraints on underlying homographies, not just homography matrices. We shall call this set of constraints the singularity constraints.

\section{Experimental validation}

We explored the pertinence of the new multiplicity and singularity constraints on both synthetic and real data. Our experiments demonstrate that prevailing robust multihomography estimation methods cannot produce compatible homography matrices because they fail to enforce consistency constraints.

\subsection{Experiments on synthetic data}

We confirmed the validity of our new constraints on synthetic data by estimating multiple homographies separately with and without noise for various planar scenes (see Figure 1). Our findings conformed to theoretical expectations: with noiseless data the estimated homographies satisfied the compatibility constraints, whereas with noisy data they did not. We also evaluated our new constraints on homographies that are guaranteed to be compatible even on noisy data, namely homographies estimated using the latent variable parametrisation described in Section 2. Once again, our new constraints were satisfied in accordance with theory.

\subsection{Experiments on real data}

Extensive simulations on synthetic data reported in [5, $8,9,32,36]$ have already demonstrated that enforcing consistency constraints leads to more accurate homography estimates. Therefore rather than repeat similar simulations, we instead chose to investigate whether compatibility constraints are violated on typical real-world datasets. We decided on the AdelaideRMF dataset ${ }^{1}$ because it contains multiple planar scenes and because it is starting to receive widespread adoption by researchers who investigate robust multi-structure fitting methods $[21,26,30,35]$. The AdelaideRMF dataset consists of image pairs and manually labelled keypoint correspondences which were obtained by

\footnotetext{
${ }^{1}$ http://cs.adelaide.edu.au/ $\sim$ hwong/doku.php?id=data
} 
Table 1: Evaluation of homography compatibility constraints.

\begin{tabular}{|c|c|c|c|c|c|c|}
\hline \multirow[b]{2}{*}{ Data } & \multicolumn{2}{|c|}{ Homography Constraints } & \multicolumn{4}{|c|}{ Fundamental Matrix Fidelity } \\
\hline & Multiplicity Constr. & Singularity Constr. & Worst Smps. Dist. & Best Smps. Dist. & Consist. Smps. Dist. & Optim. Smps. Dist. \\
\hline elderhall & $8.84 \times 10^{-6}$ & $\mathrm{n} / \mathrm{a}$ & $2.7811 \times 10^{4}$ & $2.5113 \times 10^{4}$ & $2.98703 \times 10^{1}$ & $1.9118 \times 10^{1}$ \\
\hline ladysymon & $2.9554 \times 10^{-7}$ & $\mathrm{n} / \mathrm{a}$ & $7.71698 \times 10^{1}$ & $7.14105 \times 10^{1}$ & $6.79467 \times 10^{1}$ & $6.6813 \times 10^{1}$ \\
\hline library & $8.4870 \times 10^{-9}$ & $\mathrm{n} / \mathrm{a}$ & $7.914586 \times 10^{2}$ & $1.756249 \times 10^{2}$ & $6.65369 \times 10^{1}$ & $5.6452 \times 10^{1}$ \\
\hline nese & $3.7071 \times 10^{-8}$ & $\mathrm{n} / \mathrm{a}$ & $7.63864 \times 10^{1}$ & $6.49791 \times 10^{1}$ & $6.26723 \times 10^{1}$ & $6.2645 \times 10^{1}$ \\
\hline sene & $6.7779 \times 10^{-8}$ & $\mathrm{n} / \mathrm{a}$ & $5.70514 \times 10^{1}$ & $3.56574 \times 10^{1}$ & $3.39553 \times 10^{1}$ & $3.3911 \times 10^{1}$ \\
\hline napiera & $3.1705 \times 10^{-7}$ & $\mathrm{n} / \mathrm{a}$ & $3.151676 \times 10^{2}$ & $2.87451 \times 10^{1}$ & $2.34959 \times 10^{1}$ & $1.7609 \times 10^{1}$ \\
\hline hartley & $5.7434 \times 10^{-8}$ & $\mathrm{n} / \mathrm{a}$ & $1.08842 \times 10^{2}$ & $1.051076 \times 10^{2}$ & $1.047488 \times 10^{2}$ & $1.0452 \times 10^{2}$ \\
\hline oldclassicwings & $3.6707 \times 10^{-8}$ & $\mathrm{n} / \mathrm{a}$ & $1.597191 \times 10^{2}$ & $1.580794 \times 10^{2}$ & $1.546744 \times 10^{2}$ & $1.4819 \times 10^{2}$ \\
\hline barrsmith & $2.3877 \times 10^{-8}$ & $\mathrm{n} / \mathrm{a}$ & $1.0274 \times 10^{3}$ & $3.599146 \times 10^{2}$ & $9.58709 \times 10^{1}$ & $9.407 \times 10^{1}$ \\
\hline neem & $4.2163 \times 10^{-5}$ & $2.2430 \times 10^{4}$ & $2.8117 \times 10^{4}$ & $8.440351 \times 10^{2}$ & $6.390307 \times 10^{2}$ & $5.8102 \times 10^{2}$ \\
\hline elderhallb & $4.7339 \times 10^{-6}$ & $8.1410 \times 10^{-8}$ & $1.3215 \times 10^{4}$ & $5.43782 \times 10^{1}$ & $4.49781 \times 10^{1}$ & $4.3403 \times 10^{1}$ \\
\hline napierb & $7.8316 \times 10^{-6}$ & $1.6831 \times 10^{11}$ & $3.6515 \times 10^{3}$ & $6.713961 \times 10^{2}$ & $6.506059 \times 10^{2}$ & $6.3202 \times 10^{2}$ \\
\hline johnsona & $1.8434 \times 10^{-6}$ & $3.7940 \times 10^{-1}$ & $5.5611 \times 10^{5}$ & $1.3717 \times 10^{3}$ & $1.2884 \times 10^{3}$ & $1.2891 \times 10^{3}$ \\
\hline johnsonb & $1.0 \times 10^{-3}$ & $1.3745 \times 10^{5}$ & $2.9557 \times 10^{6}$ & $4.1169 \times 10^{3}$ & $3.9156 \times 10^{3}$ & $3.9123 \times 10^{3}$ \\
\hline bonhall & $9.1052 \times 10^{-7}$ & $1.4353 \times 10^{8}$ & $8.6778 \times 10^{6}$ & $2.590761 \times 10^{2}$ & $1.010674 \times 10^{2}$ & $1.0096 \times 10^{2}$ \\
\hline
\end{tabular}

Evaluation of homography compatibility constraints on the AdelaideRMF datasets, where each homography was estimated separately using DLT and refined using separate bundle adjustment. Homographies were estimated from ground truth inlier sets. The results tabulated under the homography constraints heading show that the estimated homographies are mutually incompatible. Under the heading of fundamental matrix fidelity we report, for each scene, the best and worst fundamental matrix by recording the lowest and highest Sampson distance respectively. As a point of reference, we also report the fundamental matrix that can be constructed from the latent variables used to guarantee all homography consistency constraints in the work of Szpak et al. [32], as well as the globally optimal fundamental matrix using the method of Zheng et al. [37]. In line with expectations, the fundamental matrix constructed from a set of mutually compatible homography matrices is substantially more accurate than the best possible fundamental matrix constructed from incompatible homographies. For most scenes the fundamental matrix associated with compatible homography matrices can be identified with the globally optimal fundamental matrix.

SIFT matching, as well as randomly generated incorrect matches (outliers) - see Figure 2. Because the purpose of robust multi-structure fitting methods is to find all inliers corresponding to all structures, we hypothesised the existence of a method that achieves this goal and examined whether estimating homographies separately whilst utilising all inlying structures would result in a compatible set of homography matrices.

After establishing sets of inliers for various structures, most robust multi-homography estimation methods use the direct linear transform (DLT) to estimate initial homographies, and refine them with separate bundle adjustment. We adopted the same protocol and estimated a set of homography matrices for each AdelaideRMF scene. We then evaluated our new consistency constraints on the homography matrices. The results shown in Table 1 under the heading homography constraints indicate that the homographies obtained were incompatible.

The consequence of homographies being incompatible can be further appreciated by examining the extent to which a fundamental matrix constructed from an incompatible set of homographies can represent the epipolar geometry of the scene. As is well known, given a homography $\mathbf{H}$ between the first and second views induced by a plane in the scene, the underlying fundamental matrix $\mathbf{F}$ can be written as

$$
\mathbf{F}=\mathbf{H}^{-\top}[\mathbf{e}]_{\times},
$$

where e represents the epipole in the first image. ${ }^{2}$ As stated in Section 5, the epipole in the first image is a fixed point of any homology with matrix of the form $\mathbf{H}_{j}^{-1} \mathbf{H}_{i}$ and can be retrieved as the eigenvector corresponding to a non-repeated eigenvalue of any of the $\mathbf{H}_{j}^{-1} \mathbf{H}_{i}$ 's. If the homography matrices $\mathbf{H}_{i}$ are incompatible, the various homology matrices $\mathbf{H}_{j}^{-1} \mathbf{H}_{i}$ will also be incompatible and will lead to different estimates of the epipole in the first image. This in turn will lead to different estimates of the fundamental matrix (see Figure 3 for an example). Moreover, if a fundamental matrix is constructed by selecting a particular homography matrix $\mathbf{H}_{i_{0}}$ in (12), then even though the resulting fundamental matrix may be compatible with $\mathbf{H}_{i_{0}}$, it will not be compatible with the remaining homographies $\mathbf{H}_{i}, i \neq i_{0}$. Hence, given multiple incompatible homographies, it is possible to construct multiple incompatible fundamental matrices.

It is customary to evaluate the fidelity of a fundamental matrix by evaluating the Sampson distance on the set of corresponding points. The Sampson distance yields a measure of how accurately a fundamental matrix represents the epipolar geometry of the scene. To demonstrate the vagaries of constructing fundamental matrices from incompatible homographies, we composed all possible fundamental

\footnotetext{
${ }^{2}$ The standard formula for $\mathbf{F}$ in terms of $\mathbf{H}$ is $\mathbf{F}=\left[\mathbf{e}^{\prime}\right]_{\times} \mathbf{H}$, where $\mathbf{e}^{\prime}$ stands for the epipole in the second image [17, Corollary 13.4]. From this, the alternative representation (12) readily follows by noting that $\mathbf{e}^{\prime}=\mathbf{H e}$, $[\mathbf{H e}]_{\times}=(\operatorname{det} \mathbf{H}) \mathbf{H}^{-\top}[\mathbf{e}]_{\times} \mathbf{H}^{-1}[3$, Fact 3.10.1, xxxvi)], and further by dropping the irrelevant scalar factor $\operatorname{det} \mathbf{H}$.
} 


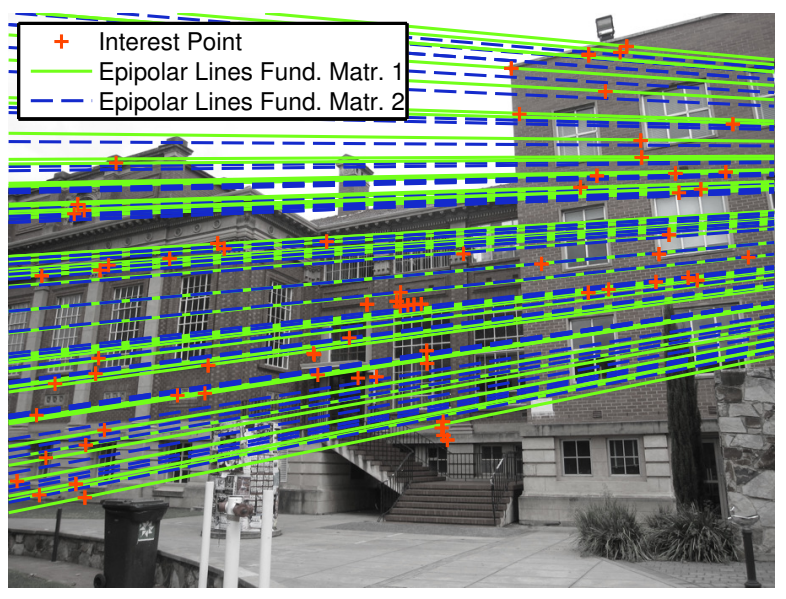

Figure 3: Comparison of epipolar lines associated with two fundamental matrices computed from two separately estimated homographies on the barrsmith dataset. The epipolar lines associated with the two fundamental matrices do not overlap, and thereby demonstrate that the two homographies do not share the same epipolar geometry and hence are incompatible.

matrices and reported the best and worst fundamental matrix in terms of the Sampson distance measure. The results recorded in Table 1 under the heading fundamental matrix fidelity unequivocally indicate that a fundamental matrix constructed from consistent homography matrices using equation (5) is superior to the best fundamental matrix constructed from inconsistent homographies. As a further point of reference, we also report the Sampson distance associated with the globally optimal fundamental matrix which we computed using the branch and contract algorithm described in the work of Zheng et al. [37]. Remarkably, for most scenes in the AdelaideRMF dataset, the fundamental matrix associated with consistent homography matrices can be identified with the globally optimal fundamental matrix.

\section{Discussion}

Various researchers over the years have espoused the importance of enforcing consistency constraints in multiple view geometry [2, 14, 15, 24, 27, 28, 33]. Nevertheless, the predominant practice in robust projective multi-structure estimation is to ignore constraints. There are several reasons why constraints are often not enforced. Part of the problem is a lack of awareness that constraints exist even in the general non-Euclidean setting (i.e. with uncalibrated cameras). Furthermore, in some contexts, such as multiple homography estimation, a full set of constraints is yet to be discovered and simple reliable constrained estimation methods still need to be developed.

We have argued that knowledge of explicit homography constraints is of practical relevance for two reasons: (1) if explicit constraints were known, they could be enforced using a non-linear constrained optimisation procedure, resulting in a new multi-homography estimation method; and (2) the compatibility of homography matrices can be ascertained by evaluating the constraints. Despite these benefits, the utility of the derived constraints has been challenged by some of our peers under the premise that the difference in residual error between homographies with consistency constraints enforced, versus homographies where consistency is not enforced, can serve as an indicator for how compatible homographies are. The tacit assumption is that smaller residuals imply more compatible homographies. This assumption is incorrect. In fact, the residual error for homographies with consistency enforced will be equal to or larger than that for homographies without consistency enforced. This follows from the general principle that an unconstrained optimum has a lower value of the underlying cost function than a constrained one. Hence, the residual error cannot be used to make statements about the compatibility of sets of homographies. However, just because the residual error for consistent homographies may be slightly higher does not mean that consistent homographies are less accurate. On the contrary, experiments conducted in $[8,9,32]$ demonstrate that enforcing consistency results in substantial improvements in accuracy. The apparent contradiction can be resolved with recourse to the machine learning concepts of training error-in our case, the geometric error for the set of corresponding points on which the homography is estimated-and generalisation error-in our case, the geometric error evaluated on other corresponding points that belong to the same planar structure. Enforcing homography constraints may increase the training error, but will as a rule decrease the generalisation error.

\section{Conclusion}

Through experiments on real data we have demonstrated that consistency constraints on homography matrices do matter. We have also presented and validated two new sets of explicit constraints which we believe provide theoretical foundations that facilitate the derivation of a full set of constraints. We hope that the results and arguments presented in this paper will trigger a new stream of research in robust projective multi-structure estimation. We anticipate a new generation of estimation methods that enforce full consistency constraints on sets of estimable projective entities such as fundamental and homography matrices.

\section{Acknowledgements}

This research was supported by the Australian Research Council. 


\section{References}

[1] S. Baker, A. Datta, and T. Kanade. Parameterizing homographies. Tech. Rep. CMU-RI-TR-06-11, Robotics Institute, Carnegie Mellon University, Pittsburgh, PA, 2006. 2

[2] A. Bartoli and P. Sturm. Constrained structure and motion from multiple uncalibrated views of a piecewise planar scene. Int. J. Computer Vision, 52(1):45-64, 2003. 8

[3] D. S. Bernstein. Matrix Mathematics: Theory, Facts, and Formulas. Princeton University Press, Princeton, NJ, 2nd edition, 2009. 7

[4] J. Bochnak, M. Coste, and M.-F. Roy. Real Algebraic Geometry. Springer, Berlin, 1998. 3

[5] P. Chen and D. Suter. Rank constraints for homographies over two views: revisiting the rank four constraint. Int. J. Computer Vision, 81(2):205-225, 2009. 1, 6

[6] T.-J. Chin, H. Wang, and D. Suter. The ordered residual kernel for robust motion subspace clustering. In Adv. Neural Inf. Process. Systems, volume 22, pages 333-341. 2009. 1

[7] T.-J. Chin, H. Wang, and D. Suter. Robust fitting of multiple structures: the statistical learning approach. In Proc. 12th Int. Conf. Computer Vision, pages 413-420, 2009. 1

[8] W. Chojnacki, Z. Szpak, M. J. Brooks, and A. van den Hengel. Multiple homography estimation with full consistency constraints. In Proc. Int. Conf. Digital Image Computing: Techniques and Applications, pages 480-485, 2010. 2, 3, 6, 8

[9] W. Chojnacki, Z. L. Szpak, M. J. Brooks, and A. van den Hengel. Enforcing consistency constraints in uncalibrated multiple homography estimation using latent variables. Mach. Vision Appl., 26(2):401-422, 2015. 2, 3, 6, 8

[10] W. Chojnacki and A. van den Hengel. A dimensionality result for multiple homography matrices. In Proc. 13th Int. Conf. Computer Vision, pages 2104-2109, 2011. 1, 3

[11] W. Chojnacki and A. van den Hengel. On the dimension of the set of two-view multi-homography matrices. Complex Anal. Oper. Theory, 7(2):465-484, 2013. 1, 3

[12] M. Decrouez, R. Dupont, F. Gaspard, and J. L. Crowley. Extracting planar structures efficiently with revisited BetaSAC. In Proc. 21st Int. Conf. Pattern Recognition, pages 21002103, 2012. 1

[13] D. F. Fouhey, D. Scharstein, and A. J. Briggs. Multiple plane detection in image pairs using J-linkage. In Proc. 20th Int. Conf. Pattern Recognition, pages 336-339, 2010. 1

[14] J. Goldberger. Reconstructing camera projection matrices from multiple pairwise overlapping views. Comput. Vis. Image Underst., 97(3):283-296, 2005. 8

[15] V. M. Govindu. Combining two-view constraints for motion estimation. In Proc. IEEE Conf. Computer Vision and Pattern Recognition, volume 2, pages 218-225, 2001. 8

[16] J. Harris. Algebraic Geometry: A First Course. Springer, New York, 1995. 2

[17] R. I. Hartley and A. Zisserman. Multiple View Geometry in Computer Vision. Cambridge University Press, Cambridge, 2nd edition, 2004. 1, 3, 7

[18] R. S. Irving. Integers, Polynomials, and Rings: A Course in Algebra. Springer, New York, 2004. 4
[19] H. Isack and Y. Boykov. Energy-based geometric multimodel fitting. Int. J. Computer Vision, 97(2):123-147, 2012. 1

[20] M. Kirchhof. Linear constraints in two-view multiple homography estimation of uncalibrated scenes. In Int. Arch. Photogrammetry, Remote Sensing and Spatial Inform. Sciences, volume XXXVII-B3a, pages 13-20, 2008. 2

[21] K. H. Lee and S. W. Lee. Deterministic fitting of multiple structures using iterative MaxFS with inlier scale estimation. In Proc. 14th Int. Conf. Computer Vision, pages 41-48, 2013. 6

[22] G. López-Nicolás, J. J. Guerrero, O. A. Pellejero, and C. Sagüés. Computing homographies from three lines or points in an image pair. In Proc. Int. Conf. Image Analysis and Processing, volume 3617 of Lecture Notes in Computer Science, pages 446-453. Springer, 2005. 2

[23] H. Lütkepol. Handbook of Matrices. John Wiley \& Sons, Chichester, 1996. 2

[24] Y. Ma, K. Huang, R. Vidal, J. Košecká, and S. Sastry. Rank conditions on the multiple-view matrix. Int. J. Computer Vision, 59(23):115-137, 2004. 8

[25] Y. Ma, S. Soatto, J. Košecká, and S. Sastry. An Invitation to 3-D Vision: From Images to Geometric Models. Springer, New York, 2nd edition, 2005. 2

[26] L. Magri and A. Fusiello. T-linkage: a continuous relaxation of J-linkage for multi-model fitting. In Proc. IEEE Conf. Computer Vision and Pattern Recognition, pages 39543961, 2014. 6

[27] E. Malis and R. Cipolla. Multi-view constraints between collineations: application to self-calibration from unknown planar structures. In Proc. 6th European Conf. Computer Vision, volume 1843 of Lecture Notes in Computer Science, pages $610-624,2000.8$

[28] J. Meidow, W. Föster, and C. Beder. Optimal parameter estimation with homogeneous entities and arbitrary constraints. In Proc. 31st DAGM Symposium, volume 5748 of Lecture Notes in Computer Science, pages 292-301, 2009. 8

[29] S. Mittal, S. Anand, and P. Meer. Generalized projectionbased M-estimator. IEEE Trans. Pattern Anal. Mach. Intell., 34(12):2351-2364, Dec. 2012. 1

[30] T. T. Pham, T.-J. Chin, J. Yu, and D. Suter. The random cluster model for robust geometric fitting. IEEE Trans. Pattern Anal. Mach. Intell., 36:1658-1671, 2014. 1, 6

[31] A. Shashua and S. Avidan. The rank 4 constraint in multiple ( $\geq 3$ ) view geometry. In Proc. 4th European Conf. Computer Vision, volume 1065 of Lecture Notes in Computer Science, pages 196-206, 1996. 1

[32] Z. L. Szpak, W. Chojnacki, A. Eriksson, and A. van den Hengel. Sampson distance based joint estimation of multiple homographies with uncalibrated cameras. Comput. Vis. Image Underst., 125:200-213, 2014. 2, 3, 5, 6, 7, 8

[33] T. Ueshiba and F. Tomita. Plane-based calibration algorithm for multi-camera systems via factorization of homography matrices. In Proc. 9th Int. Conf. Computer Vision, volume 2, pages $966-973,2003.8$

[34] H. Wang, T.-J. Chin, and D. Suter. Simultaneously fitting and segmenting multiple-structure data with outliers. IEEE 
Trans. Pattern Anal. Mach. Intell., 34(6):1177-1192, 2012.

1

[35] H. S. Wong, T.-J. Chin, J. Yu, and D. Suter. Dynamic and hierarchical multi-structure geometric model fitting. In Proc. 13th Int. Conf. Computer Vision, pages 1044-1051, 2011. 1, 6

[36] L. Zelnik-Manor and M. Irani. Multiview constraints on homographies. IEEE Trans. Pattern Anal. Mach. Intell., 24(2):214-223, 2002. 1, 6

[37] Y. Zheng, S. Sugimoto, and M. Okutomi. A branch and contract algorithm for globally optimal fundamental matrix estimation. In Proc. IEEE Conf. Computer Vision and Pattern Recognition, pages 2953-2960, 2011. 3, 7, 8

[38] Y. Zheng, S. Sugimoto, and M. Okutomi. A practical rankconstrained eight-point algorithm for fundamental matrix estimation. In Proc. IEEE Conf. Computer Vision and Pattern Recognition, pages 1546-1553, 2013. 3

[39] M. Zuliani, C. S. Kenney, and B. S. Manjunath. The multiRANSAC algorithm and its application to detect planar homographies. In Proc. Int. Conf. Image Processing, volume 3, pages 153-156. IEEE, 2005. 1 